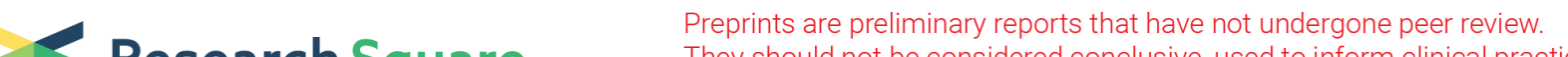 Research Square They should not be considered conclusive, used to inform clinical practice, or referenced by the media as validated information.
}

\section{Clinical Insights into Cisplatin-Induced Arrhythmia in a Patient with Locally Advanced Non-Small Cell Lung Cancer: A Case Report}

\section{Yan Huang}

West China Hospital of Sichuan University

\section{Fengzhu Guo}

West China Hospital of Sichuan University

Shuang Dai

West China Hospital of Sichuan University

Jiewei Liu

West China Hospital of Sichuan University

Feng Luo ( $\square$ luofeng66666@sina.com )

West China Hospital of Sichuan University https://orcid.org/0000-0001-6272-5005

\section{Case report}

Keywords: Lung cancer, Cisplatin, Cardiotoxicity, Arrhythmia, Cardio-Oncology

Posted Date: November 19th, 2020

DOI: https://doi.org/10.21203/rs.3.rs-108069/v1

License: (c) (i) This work is licensed under a Creative Commons Attribution 4.0 International License. Read Full License 


\section{Abstract}

Introduction: Cardiotoxicity is a common adverse effect of many antineoplastic agents including anthracyclines and paclitaxel. However, it has not been defined as a causal side effect of cisplatin.

Case report: We report a 69-year-old male with locally advanced non-small cell lung cancer who developed a cardiotoxic event induced by cisplatin that was manifested primarily as arrhythmia. The frequency and severity of the arrhythmia had a strong temporal relationship with the administration of cisplatin, which made it probable that cisplatin was responsible for the cardiotoxicity observed.

Discussion: The arrhythmias might be at least partly due to cancer pain through stimulation of the sympathetic nerves. Therefore, particular attention should be paid to patients with pre-existing cardiac disease and/or mediastinal tumors. It is important to evaluate cardiac function prior to chemotherapy and to consider cardiac monitoring during cisplatin infusions in susceptible patients with no more ECG abnormalities detected.

\section{Introduction}

Cardiac toxicity is a well-known consequence of chemotherapeutic agents and biologicals, and is predominantly seen with anthracyclines such as doxorubicin, epirubicin and daunorubicin [1]. Additionally, paclitaxel, 5-fluorouracil, methotrexate and targeted drugs (eg trastuzumab, bevacizumab and lapatinib) can also lead to myocardial damage [2, 3]. Acute cardiotoxic reactions are manifested mainly as sinus tachycardia, arrhythmia, conduction block, ST segment decline or flattened T waves, and are often reversible after drug withdrawal and symptomatic management. Delayed cardiac dysfunction is characterized by congestive heart failure and myocardial cell damage as well as by degeneration [4]. However, cardiac complications are not established side effects of cisplatin. Here, we report on a patient with locally advanced non-small cell lung cancer who developed a cardiotoxic event induced by cisplatin that was manifested primarily as arrhythmia.

\section{Case Report}

A 69-year-old man with a history of tobacco cigarette smoking was admitted for left chest pain of five months' duration. The patient had previously presented to hospital and was diagnosed, after a spectrum of cardiac examinations, with coronary atherosclerosis and ventricular premature contraction. He was treated with pharmacotherapy to improve cardiac function and coronary circulation. However, this conventional treatment was ineffective as he continued to experience chest pain repeatedly. He was subsequently referred to our hospital for assessment. The outpatient contrast-enhanced computed tomography (CT) revealed a mass shadow located in the upper pulmonary lobe near the left hilum. Additionally, there were enlarged left hilum and mediastinal lymph nodes, chronic bronchitis, emphysema and chronic infection across both lungs (Fig. 1A-1C). Four endobronchial ultrasound (EBUS) needle aspirates were conducted to try and get a pathological diagnosis. However, these were not successful 
and a transesophageal endoscopy was performed. This enabled detection of adenocarcinoma cells from the mediastinal mass. The patient was then diagnosed as having left lung adenocarcinoma with left hilar and mediastinal lymph node metastasis (cT2aN3M0, IIIB). No gene mutation was found except for the insertion mutation in ERBB2 frame (3.81\%). The patient was inappropriate for surgery and so he was transferred to our lung cancer center for chemotherapy.

The patient's chest pain recurred before chemotherapy was commenced and a thorough examination was carried out including evaluation of heart function. An electrocardiogram (ECG) indicated sinus rhythm with ventricular premature contraction, while myocardial markers and pro-brain natriuretic peptide concentrations were within the normal limits. The ECG then returned to normal following initiation of a cardiologist's recommended treatment strategy aimed at anticoagulation, lipid-lowering and improving coronary blood flow (Fig. 2A). A first-line chemotherapy regimen of intravenous pemetrexed (840 mg on day 1 ) and cisplatin ( $45 \mathrm{mg}$ on day 1 followed by $40 \mathrm{mg}$ on days 2 and 3 ) was commenced. During chemotherapy, patients were given ECG monitoring, polarization fluid (10 IU of insulin $+10 \% \mathrm{KCL} 10 \mathrm{ml}+$ $10 \%$ glucose $500 \mathrm{ml}$ ) to stabilize the potential of myocardial cell membrane, antiemetic and analgesic drug therapy. There were no apparent adverse effects until the end of the first day of this first cycle when the patient complained of increased and persistent chest and back pain. Intensive cardiac monitoring displayed frequent ventricular premature systole without abnormal variations of heart rate (Fig. 2B). There was no significant change in the results of laboratory examinations during chemotherapy (Table 1). It was considered that his signs may be cancer-related pain. Therefore, bucinnazine $100 \mathrm{mg}$ was administered with symptom relief and a decrease in ventricular premature beats detected by ECG monitoring. The chemotherapy was continued on the second day with no more overt cardiac or chestrelated manifestations (Fig. 2C). However, on the third day, the patient experienced another episode of chest discomfort during cisplatin infusion. ECG showed a heart rhythm of $40-70$ beats per minute, ventricular premature contractions (bigeminy and trigeminy) and sinus bradycardia indicating gradual deterioration of sinus function (Fig. 2D and E). Electrolytes, myocardial markers and blood pressure did not suggest any cardiac abnormalities. The cisplatin infusion was discontinued immediately and the patient was observed. Overnight, the patient complained of more serious chest pain and was given another dose of bucinnazine, which resulted in symptom amelioration and normal sinus rhythm. An ECG was repeated the next day, with no obvious sign of cardiac arrhythmia and no reappearance of chest discomfort (Fig. 2F). Upon his second visit to hospital for chemotherapy, an ECG only showed rare ventricular premature contractions similar to those observed before his first chemotherapy. Given his last cardiac adverse event, single-agent pemetrexed was utilized with no more ECG abnormalities detected. 
Table 1

The results of laboratory examinations during chemotherapy.

\begin{tabular}{|c|c|c|c|c|c|}
\hline Time & $\begin{array}{l}\mathrm{K}^{+} \\
(\mathrm{mmol} / \mathrm{L})\end{array}$ & $\begin{array}{l}\text { Myo } \\
(\mathrm{ng} / \mathrm{ml})\end{array}$ & $\begin{array}{l}\text { Ck-MB } \\
\text { (ng/ml) }\end{array}$ & $\begin{array}{l}\text { TPN-T } \\
\text { (ng/L) }\end{array}$ & $\begin{array}{l}\text { PRO-BNP } \\
\text { (pg/ml) }\end{array}$ \\
\hline Pre-Chemotherapy & 4.21 & 36.78 & 3.83 & 17.3 & 39 \\
\hline $\begin{array}{l}\text { During } \\
\text { Chemotherapy }\end{array}$ & 4.12 & 21.12 & 1.49 & 13.7 & NA \\
\hline Post-Chemotherapy & 3.97 & 29.85 & 2.3 & 13.7 & 433 \\
\hline Reference Value & $3.5-5.3$ & $<72.0$ & $<4.94$ & $0-14$ & $0-227$ \\
\hline
\end{tabular}

\section{Discussion}

Cisplatin has a wide range of antineoplastic effects and is considered to be the foundation of doublechemotherapy for lung cancer [5]. The most frequently observed toxicities of cisplatin involve the kidneys, nervous system, ears, bone marrow and gastrointestinal tract [6]. Pemetrexed plays its anticancer role by inhibiting multiple targets of the folate metabolic pathway [7]. Both pemetrexed and cisplatin are considered to carry low risk of cardiac toxicity, which is part of the reason for choosing this regimen for our patient given his atherosclerotic heart disease. Cardiac complications are not established side effects of cisplatin. However, in cases like ours it seems probable that cisplatin was responsible for the cardiotoxicity observed. First of all, the frequency and severity of arrhythmia closely related to the continuous administration of cisplatin. Second, recovery of normal sinus rhythm was observed the day after the cisplatin infusion was discontinued. Third, compared with the first cycle, there were no newly observed arrhythmias and no deterioration in existing arrhythmias during the second cycle when cisplatin was the only discontinued agent. Based on the Naranjo et al. algorithm [8], he had a probable adverse drug reaction (Table 2). Collectively, this information clearly relates cardiac toxicity to cisplatin in this patient. 
Table 2

Naranjo scoring for patient, noting probable adverse drug reaction.

\begin{tabular}{|c|c|c|}
\hline Question & Answer & Score \\
\hline Are there previous conclusive reports on this reaction? & Yes & +1 \\
\hline Did the adverse event appear after the suspected drug was given? & Yes & +2 \\
\hline $\begin{array}{l}\text { Did the adverse reaction improve when the drug was discontinued or a } \\
\text { specific antagonist was given? }\end{array}$ & Yes & +1 \\
\hline Did the adverse reaction appear when the drug was readministered? & Yes & +2 \\
\hline Are there alternative causes that could have caused the reaction? & $\begin{array}{l}\text { Do not } \\
\text { know }\end{array}$ & 0 \\
\hline Did the reaction reappear when a placebo was given? & $\begin{array}{l}\text { Not } \\
\text { done }\end{array}$ & 0 \\
\hline Was the drug detected in any body fluid in toxic concentrations? & $\begin{array}{l}\text { Not } \\
\text { done }\end{array}$ & 0 \\
\hline $\begin{array}{l}\text { Was the reaction more severe when the dose was increased or less severe } \\
\text { when the dose was decreased? }\end{array}$ & Yes & +1 \\
\hline $\begin{array}{l}\text { Did the patient have a similar reaction to the same or similar drugs in any } \\
\text { previous exposure? }\end{array}$ & $\begin{array}{l}\text { Do not } \\
\text { know }\end{array}$ & 0 \\
\hline Was the adverse event confirmed by objective evidence? & Yes & +1 \\
\hline Total & & $\begin{array}{l}8 \\
\text { (proba }\end{array}$ \\
\hline
\end{tabular}

Cisplatin-based combinations have become one of the most widely-used chemotherapeutic regimens since cisplatin's approval by the Food and Drug Administration in the United States of America in 1979 [9]. However, limited understanding of cisplatin-induced cardiotoxicity has been illustrated [10, 11]. Over the past decade, a diverse range of cisplatin-related cardiac complications has been reported, such as myocarditis, pericarditis, angina, myocardial ischemia, and heart failure [7, 12-15]. Hypertension and hypotension have also been observed in cisplatin-evoked cardiac toxicity [16]. Arrhythmia is the most commonly noted cardiac insufficiency appearing in the form of tachycardia with cisplatin [10,17]. Cardiotoxic effects caused by cisplatin may also manifest as bradycardia or asymptomatic arrhythmias, and mostly occur during or immediately after cisplatin infusion. These may sometimes be clinically important $[4,10]$. Since administration of cisplatin is generally not conducted under cardiac monitoring, asymptomatic bradycardia may not be noticed in clinical practice. Hence, the actual incidence of cardiotoxic events ascribed to cisplatin might be underestimated [18]. The various possible mechanisms underpinning cisplatin-induced cardiac dysfunction remain under investigation [19]. Drug-induced damage to cardiac myocytes or blood vessels, the electrical conduction system, or the pericardium are possible underlying mechanisms according to existing studies $[7,20]$. Pathological mechanisms 
including induction of coronary vasospasm, damage of the vascular endothelium, oxidative and nitrosative stress, and electrolyte imbalance are frequently associated with acute complications, whereas cardiomyopathy may be the cause of late complications $[13,15,19]$. Moreover, it is published that cisplatin could deposit in tissues such as the liver, skin and kidneys and be detectable in blood 20 years after completion of a cisplatin infusion. This suggests the possibility that cisplatin could be deposited in the sinoatrial node and vessels causing long-term cardiotoxicity $[18,21]$. Although complex, cardiotoxic manifestations of cisplatin are a critical issue that warrant further clarification and identification of a corrective solution [22].

In clinical practice, the possibility of cisplatin cardiotoxic effects should be taken into account. Currently, it is recommended that the total cisplatin accumulation it controlled to prevent high-risk drug exposure. Our patient had high sensitivity to cardiotoxicity as a result of his prior history of heart disease (coronary heart disease and arrhythmia), which made him more vulnerable to cardiotoxic drugs. Additionally, tumor metastasis to the hilar and mediastinal lymph nodes adjacent to the heart may partly explain the cause of cardiac symptoms such as chest pain and potentially complicate the differential diagnosis of the cardiac syndrome. As the patient experienced a decrease in the frequency of premature ventricular beats and relief of other arrhythmias after administration of an analgesic, there was speculation that the arrhythmias might be at least partly due to cancer pain through stimulation of the sympathetic nerves. In patients, like ours, with risk factors for cardiotoxicity, it is prudent to utilize chemotherapeutic agents with potential cardiotoxic effects cautiously and to consider ECG monitoring during chemotherapy if use is unavoidable.

In summary,while platinum-based dual therapy is the cornerstone of chemotherapy for non-small cell lung cancer, cisplatin-induced cardiotoxicity should not be overlooked. Thereforelparticular attention should be paid to patients with pre-existing cardiac disease and/or mediastinal tumors. It is important to evaluate cardiac function prior to chemotherapy and to consider cardiac monitoring during cisplatin infusions in susceptible patients. Further, use of potentially cardiotoxic concomitant medications, such as ondansetron and tricyclic antidepressants, which might induce QT interval prolongation should ideally also be avoided [23].

\section{Declarations}

\section{Ethics approval and consent to participate:}

Not applicable.

\section{Consent for publication:}

Not applicable, because the images are entirely unidentifiable and there are no details on individual reported within the manuscript.

\section{Availability of data and materials:}


Not applicable, because the images are entirely unidentifiable and there are no details on individual reported within the manuscript.

\section{Competing interests:}

The author(s) declared no potential conflicts of interest with respect to the research, authorship, and/or publication of this article.

\section{Funding:}

The authors received no financial support for the research, authorship, and/or publication of this article.

\section{Authors' contributions:}

All authors listed have made a substantial, direct and intellectual contribution to the work, and approved it for publication.

\section{Acknowledgments:}

We thank the patient and his family for providing informed consent for publication of this case.

\section{References}

1. Darling HS. Cisplatin induced bradycardia. International journal of cardiology. 2015;182:304-6. doi: 10.1016/j.ijcard.2015.01.013.

2. Bovelli D, Plataniotis G, Roila F. Cardiotoxicity of chemotherapeutic agents and radiotherapy-related heart disease: ESMO Clinical Practice Guidelines. Annals of oncology : official journal of the European Society for Medical Oncology. 2010;21 Suppl 5:v277-82. doi: 10.1093/annonc/mdq200.

3. Polk A, Vaage-Nilsen M, Vistisen K, Nielsen DL. Cardiotoxicity in cancer patients treated with 5fluorouracil or capecitabine: a systematic review of incidence, manifestations and predisposing factors. Cancer treatment reviews. 2013;39(8):974-84. doi: 10.1016/j.ctrv.2013.03.005.

4. Kucharz J, Michalowska-Kaczmarczyk A, Zygulska AL, Wojtak J, Pawlik W, Herman RM, et al. Bradycardia as a rare symptom of cisplatin cardiotoxicity: A case report. Oncology letters. 2016;11(3):2297-9. doi: 10.3892/ol.2016.4195.

5. Dasari S, Bernard Tchounwou PJEJoP. Cisplatin in cancer therapy: molecular mechanisms of action. 2014;740:364-78.

6. Patanè, Cardiology SJIJo. Cardiotoxicity: Cisplatin and long-term cancer survivors. 2014;175(1):2012.

7. Su SF, Liu LF, Geng YC, Ouyang WW, Ma Z, Li QS, et al. Pemetrexed plus cisplatin versus docetaxel plus cisplatin for stage IV lung adenocarcinoma based on propensity score matching. 2019;30(3):1-.

8. A method for estimating the probability of adverse drug reactions. \%J Clinical Pharmacology \& Therapeutics. 1981;30. 
9. Wheate NJ, Walker S, Craig GE, Oun RJDT. The status of platinum anticancer drugs in the clinic and in clinical trials. 2010;39.

10. Oun R, Rowan E. Cisplatin induced arrhythmia; electrolyte imbalance or disturbance of the SA node? European journal of pharmacology. 2017;811:125-8. doi: 10.1016/j.ejphar.2017.05.063.

11. El-Awady ESE, Moustafa YM, Abo-Elmatty DM, Radwan AJEJoP. Cisplatin-induced cardiotoxicity: Mechanisms and cardioprotective strategies. 2011;650(1):335-41.

12. Nicholas G, Kounis, Gianfranco, Cervellin, Giuseppe, cardiology LJljo. Cisplatin-induced bradycardia: Cardiac toxicity or cardiac hypersensitivity and Kounis syndrome? 2016.

13. Dugbartey GJ, Peppone LJ, Graaf IAMDJT. An integrative view of cisplatin-induced renal and cardiac toxicities: molecular mechanisms, current treatment challenges and potential protective measures. 2016;371:58-66.

14. Ryberg MJASoCOEB. Recent advances in cardiotoxicity of anticancer therapies. 2012;32(32):555.

15. Altunda? Z, ?elik I, Kars AJAoP. Recurrent asymptomatic bradycardia episodes after cisplatin infusion. 2001;35:641.

16. Khan S, Chen CL, Brady MS, Parameswaran R, Carvajal RDJJoCO. Unstable angina associated with cisplatin and carboplatin in a patient with advanced melanoma. 2012;30(18):e163.

17. Raja W, Mir MH, Dar I, Banday MA, Ahmad IJIJoM, Medical POOJolSo, et al. Cisplatin induced paroxysmal supraventricular tachycardia. 2014;34(4):330-2.

18. Schierl, Gerl A, Biology RJARTP. Urinary Excretion of Platinum in Chemotherapy-treated Long-term Survivors of Testicular Cancer. 2000;39(4):519-22.

19. Xing JJ, Hou JG, Liu Y, Zhang RB, Jiang S, Ren S, et al. Supplementation of Saponins from Leaves of Panax quinquefolius Mitigates Cisplatin-Evoked Cardiotoxicity via Inhibiting Oxidative StressAssociated Inflammation and Apoptosis in Mice. Antioxidants (Basel, Switzerland). 2019;8(9). doi: 10.3390/antiox8090347.

20. Mahrous A, Ibrahim, Gamal A, Bakhaat, Hany G, Tammam, et al. Cardioprotective effect of green tea extract and vitamin $\mathrm{E}$ on Cisplatin-induced cardiotoxicity in mice: Toxicological, histological and immunohistochemical studies. 2019.

21. Schlaeffer F, Tovi F, Leiberman AJDI, Pharmacy C. Cisplatin-induced bradycardia. 1983;17(12):899.

22. Alexandre JJP, Therapeutics. Anticancer drug-induced cardiac rhythm disorders: Current knowledge and basic underlying mechanisms. 2018;189:89-103.

23. Schlumbrecht MP, Hehr KJJOPP. Cisplatin-induced bradycardia and the importance of the QT interval. 2015;21(2):157-60.

\section{Figures}



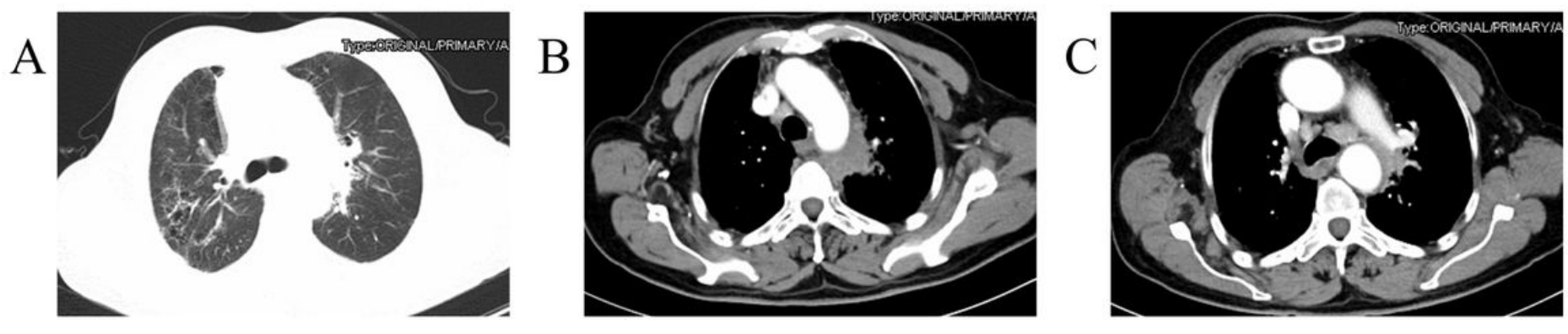

Figure 1

Representative clinical imaging findings at baseline. Chest contrast-enhanced computed tomography combined with thin-layer high-resolution scanning revealed $(A)$ a soft tissue density mass lesion of approximately $2.7 \times 4.2 \mathrm{~cm}$ near the hilum of the upper lobe of the left lung, encompassing ( $B$ and $C)$ the thoracic aorta, the left pulmonary artery and the bronchus of the left upper lobe lung, with a fuzzy boundary and stenosis of the left upper lung bronchus. The mediastinal window was enlarged with slight enhancement of multiple lymph nodes in the left hilum and mediastinum.

$\mathrm{E}$

Figure 2 
Dynamic electrocardiogram (ECG) changes before and during chemotherapy. ECG (A) before chemotherapy showed no apparent abnormality, whereas (B) arrhythmia was observed after administration of pemetrexed and cisplatin on the first day of the antitumor regimen, with the cardinal feature of ventricular premature contraction. After completion of the second day of cisplatin infusions, the ECG (C) displayed more severe ventricular premature beats and trigeminal rhythm followed by ( $D$ and E) a further deterioration of ventricular presystole immediately after the start of the cisplatin infusion the next day. This was manifested as bigeminy in association with sinus bradycardia, with a trend of gradual aggravation. Subsequently, the ECG (F) restored to sinus rhythm and no obvious ventricular premature beats existed after cisplatin was discontinued and analgesic treatment was administered. 